\title{
The Superconducting Gravimeter as a Field Instrument Applied to Hydrology
}

\author{
C.R. Wilson ${ }^{1,2}$, H. Wu ${ }^{1}$, L. Longuevergne ${ }^{1,2}$, B. Scanlon ${ }^{2}$, and J. Sharp ${ }^{1}$ \\ Department of Geological Sciences, Jackson School of Geosciences, University of Texas, Austin ${ }^{[1]}$ \\ Bureau of Economic Geology, Jackson School of Geosciences, University of Texas, Austin ${ }^{[2]}$ \\ E-mail: crwilson@mail.utexas.edu
}

\begin{abstract}
We describe development of a transportable or other subsurface fluid changes are the signal of version of the Superconducting Gravimeter (SG) and its interest.

test in a field experiment to monitor storage in a karst (limestone) aquifer in central Texas. The $\mathrm{SG}$ is contained within two aluminum enclosures, one 2 Configuration for Transport and Field holding the SG in its 35 liter helium dewar, plus
\end{abstract} electronics; and the second for refrigerator and power supply. In the field test, the SG was supported on Development began with a standard single sphere SG threaded steel rods cemented into limestone, and (serial number 047) delivered to the University of surrounded by weather-protecting sheds. The steel rod Texas in 2007. SG047 components were packaged design was not completely satisfactory, and in most within Enclosures 1 and 2 (E1 and E2), shown in field settings a concrete floor will probably be required. Figures 1 and 2. Each is constructed of aluminum, Field operation requires wired electric power, but is with dimensions $\sim 1.5 \times 0.8 \times 1 \mathrm{~m}$, and mass $\sim 250 \mathrm{~kg}$ managed remotely using wireless internet. The including SG equipment. The two enclosures provide experiment south of Austin Texas was designed to physical protection, facilitate control of temperature monitor ground water level, precipitation, and other and humidity, and simplify handling during transport. variables, and observe mass variations associated with Figure 1 shows E1 with refrigerator compressor, storage changes in the aquifer. Drought conditions power supply (UPS), and lightning protector. Figure 2 prevailed, limiting conclusions about the aquifer, but shows E2 with dewar, refrigerator cold head frame, the experiment demonstrated the feasibility of remote and electronics (data logger, levitation and sensor unattended operation for periods of many months.

Keywords. Superconducting gravimetry, hydrology controls, barometer, GPS receiver, power supply, and computer). E1 and E2 are connected by electric power lines, data cables, and helium refrigeration hoses, and are separable by up to $10 \mathrm{~m}$. In a field setting, a weather station connects to E2 and provides a mast for the system timing GPS antenna.

The Superconducting Gravimeter (SG) consists of a niobium $2.54 \mathrm{~cm}$ spherical proof mass, levitated in magnetic fields, and contained within a liquid helium bath in a dewar (Prothero and Goodkind, 1968). Early papers (Prothero and Goodkind, 1972) drew interest within the geophysics community, and led to establishment of a company (GWR) to undertake commercial production. Goodkind (1999) reviews SG principles, and a summary of history, design, performance, data analysis, and applications is given by Hinderer et al (2007). GWR gives precision for current SG's as $0.01 \mu$ gals $\left(0.1 \mathrm{~nm} \mathrm{~s}^{-2}\right)$ or better, and drift below $1 \mu$ gal $\left(10^{-5} \mathrm{~m} \mathrm{~s}^{-2}\right)$ per month. Several dozen SG's in service (Crossley et al, 1999) have confirmed that local groundwater variations are readily detectable, though this source of gravity change is usually considered a nuisance. The motivation for developing a transportable SG has been to allow installations at locations where groundwater

E1 is cooled via a top-mounted exhaust fan and requires ambient temperatures below $35^{\circ} \mathrm{C}$ following the manufacturer's (Sumitomo Heavy Industries) specifications for refrigerator compressor operations. Temperature control requirements for E2 are similar, but heat production is much lower. During laboratory trials, we tested a $0.4 \mathrm{~kW}$ cooling unit mounted on the interior of $\mathrm{E} 2$ as a possible way to regulate temperature. However, this blowing device caused unacceptable temperature cycling, leading to oscillations in the level control system (which uses a thermal mechanism). Therefore, E2 was cooled using circulated air during the field trial. 


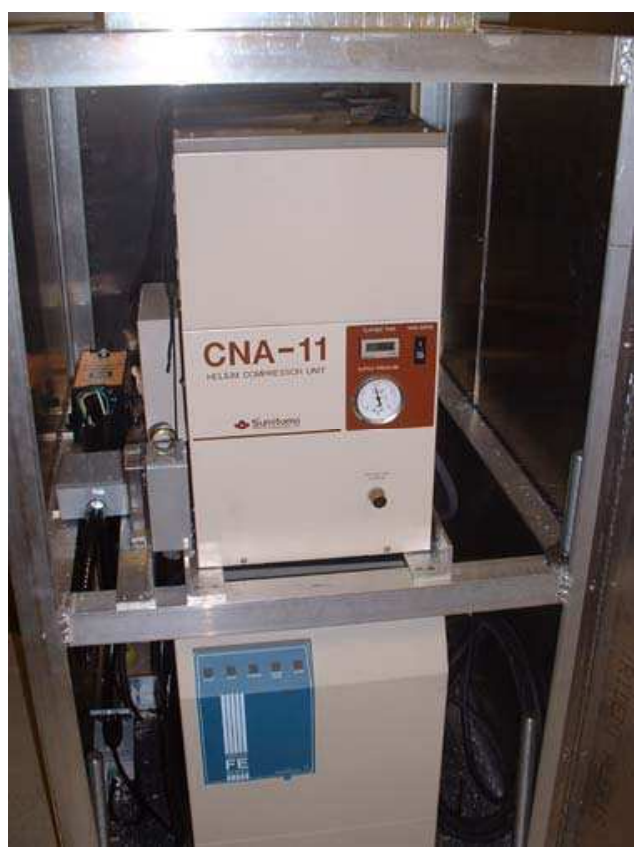

Figure 1. Interior of enclosure E1, containing UPS and refrigerator. Lightning surge protector and thermostatic control for the top-mounted cooling fan are located at midlevel.

The cold head frame is secured to the base of E2 with vibration isolation mounts, and the dewar containing the SG sensor is supported on 3 aluminum pillars. When installing on a concrete floor, the pillars are bolted to an aluminum plate. Installation involves raising E2, sliding the pillar-plate assembly beneath it, and lowering E2 as the 3 pillars pass through clearance holes to support the dewar. During the field trial, the pillar plate assembly was replaced by braced threaded rods cemented into outcropping limestone (Figure 5) with the 3 aluminum pillars threaded onto the rods.

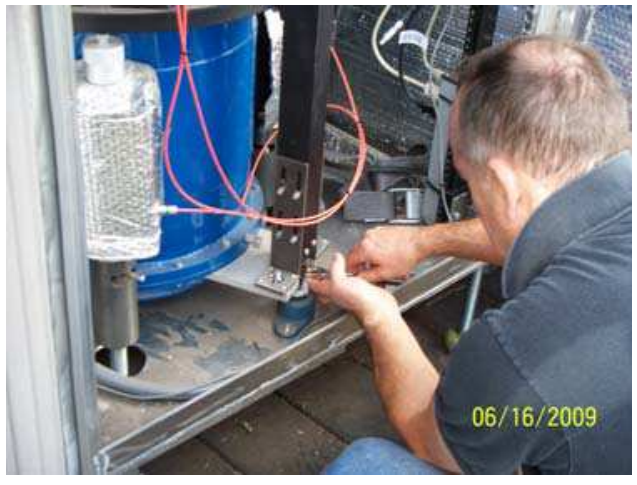

Figure 3. Securing the base of the dewar to the cold head frame with brackets allows E2 to be transported without disassembly.

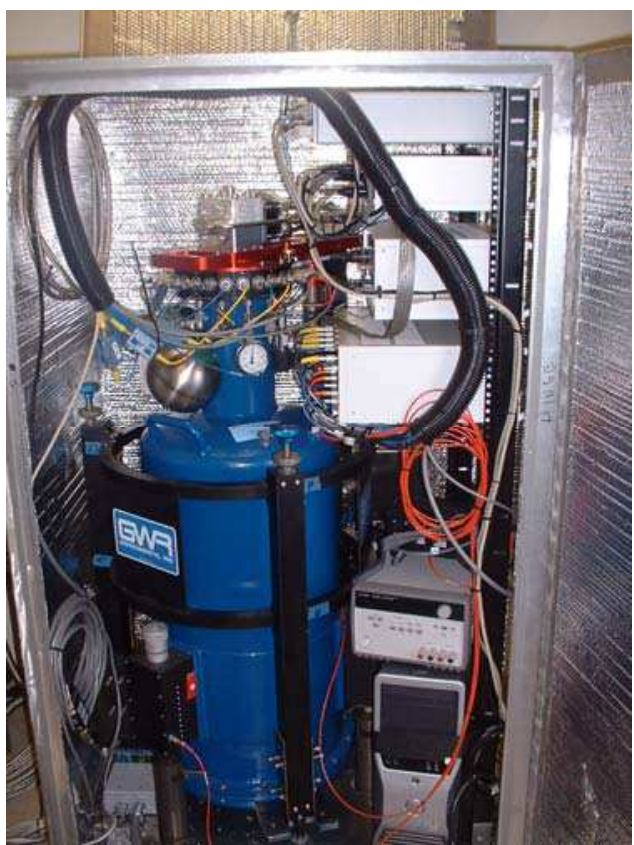

Figure 2. Interior of enclosure E2 showing helium dewar, cold head and cold head frame with rack mounting of most electronics at right.

A portable air conditioning unit supplied cool air to the base of E1, with hot air exhausted from the top E1 to the shed exterior. A fan and duct connecting the two instrument sheds circulated cool air to E1. These systems worked well, although additional reflective insulation on the exterior of the sheds was required to reduce heat load from the sun.

Relocation is accomplished with the proof mass levitated. Power cables and refrigeration lines are first disconnected. Then the dewar is attached to the base of the cold head frame using brackets shown in Figure 3. The cold head lifting arm can also be secured to the dewar neck using 4 machine screws, giving resistance to lateral motion at the top of the dewar during transport, while the lower brackets (Figure 3 ) bear the weight. After securing the dewar, E2 is raised above the aluminum pillars with hand lifts and the pillars are removed. Then E2 is lowered and secured to a wheeled dolly, and moved to the new location. After relocation the proof mass is re-centered to adjust for gravity differences at the two sites, and any helium lost in transit (while the refrigerator is off) is replenished by liquefying gas from a cylinder.

\section{Laboratory and Field Trials}

Figure 4 shows four time series of residual gravity variations over the period May 2007 to June 2009, taken during three laboratory and one field trial. Tidal 
variations, pole tide, and atmospheric mass effects were removed from all series. The first period (MayJuly 2007) (University of Texas Geology Building), shows a very high drift rate, and SG047 was returned to GWR for repairs after this. The second period (October 2007-March 2008) (University of Texas Geology Building) shows that after repairs, drift rate was reduced to $\sim 10 \mu$ gals per month, still a high rate. The third period (March - October 2008 at the University of Texas Research Campus) followed a test of relocation procedures, and included integration of the weather station. The fourth period (November 2008-June 2009) shows data from the field site south of Austin, Texas (Figures 5,6). This site is in the recharge zone of the Edwards Aquifer, adjacent to a well with a level recorder. Wireless internet allowed remote monitoring and data transfer. No permanent structure was permitted at the site, requiring the monument design shown in Figure 5.

The Edwards Aquifer (EA) is a major water resource both for the City of San Antonio, and regions to the north and west. As a karst (limestone) aquifer, groundwater is stored in small pores, fractures, and dissolution features (voids, tunnels, caves) that are spatially heterogeneous and not accessible to observation. There is no direct way to assess the volume of water stored in this aquifer. However, when both a well level record and precise observations of gravity at the surface are available, a reliable estimate of effective porosity (specific yield), should be possible. Observations of the EA in most locations show that periods of heavy rainfall cause water levels in wells to increase rapidly, by $10 \mathrm{~m}$ or more over periods of few days to a few weeks. An event of this magnitude might increase gravity by $\sim 20-40 \mu$ gals, and be detectable as a transient event, even with a large SG drift rate. It was hoped that at least one major recharge event would occur during the field trial, but the entire period was one of exceptional drought, with no significant water level changes.

Although little was learned about EA hydrologic properties the field trial provided a useful test of operations. Constraints on monument design were revealed by two major anomalies in the time series (Figure 4). The first, appearing soon after installation in November, 2008, was caused by a sagging wooden floor in the instrument shed (Figure 6), allowing the cold head frame to touch the dewar neck. Manual adjustment of the cold head frame solved this problem. (The problem also arose, to a lesser degree in May 2009). The second anomaly, in March 2009, followed a small rainfall event, insufficient to recharge the aquifer, but sufficient to wet clay-filled joints in the limestone. The joints expanded causing the monument (Figure 5) to tilt beyond the range of automatic compensation. A site visit was required to re-level. Both problems would be resolved if a poured concrete floor had been in place, and this appears to be a requirement for most field installations. Intercomparison with regular spring gravimeters highlights the high stability of the superconducting instrument with respect to temperature variations (up to $15^{\circ} \mathrm{C}$ in the shed).

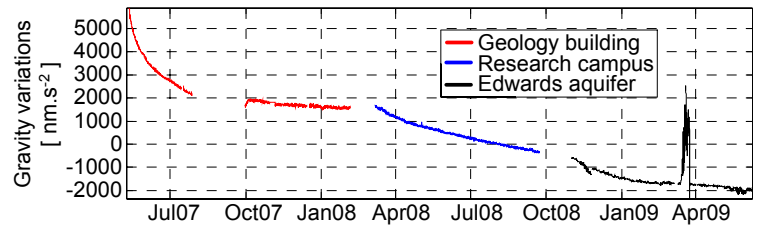

Figure 4. Four time series of SG047 residual gravity for 3 laboratory and 1 field trial from May, 2007 to June 2009. Voltage was converted to gravity using a calibration factor estimated from earth tides and a-priori tidal loading from FES2004. For hydrologic applications of the SG, a more precise calibration method (Van Camp et al, 2000) is not essential, as it typically differs from a tidal calibration by less than 1 percent. Predicted tides, tidal loading and local barometric pressure effects [admittance $-3.3 \mathrm{~nm} \mathrm{sec}^{-2} \mathrm{mbar}^{-1}$ ] were removed. The text describes the four separate time series. The last time series is from the Edwards Aquifer field trial.

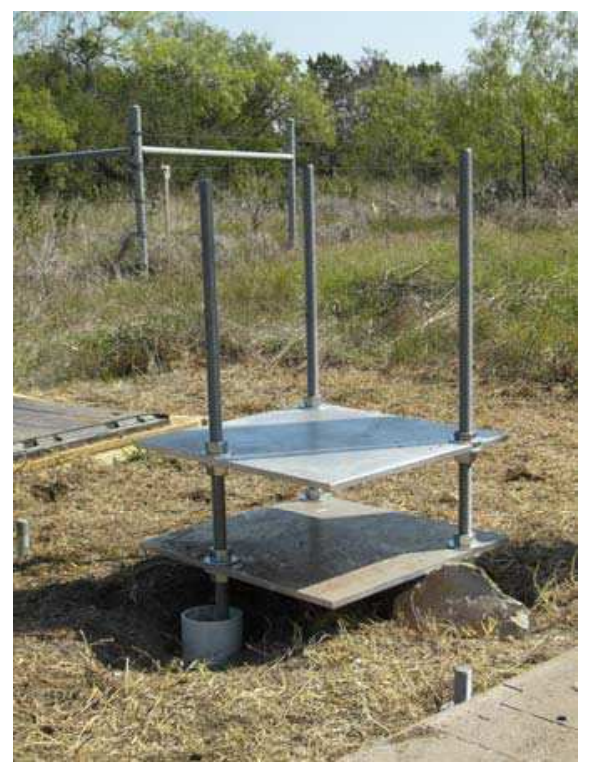

Figure 5. Edwards Aquifer field site monument installation. Plates and long rods were removed after the epoxy cement had cured. Shorter rods were installed and the plates were replaced to form bracing for the vertical rods. Finally, aluminum pillars were threaded to the top of the steel rods, and E2 lowered onto them. The last step involved erecting the instrument shed around E2. 


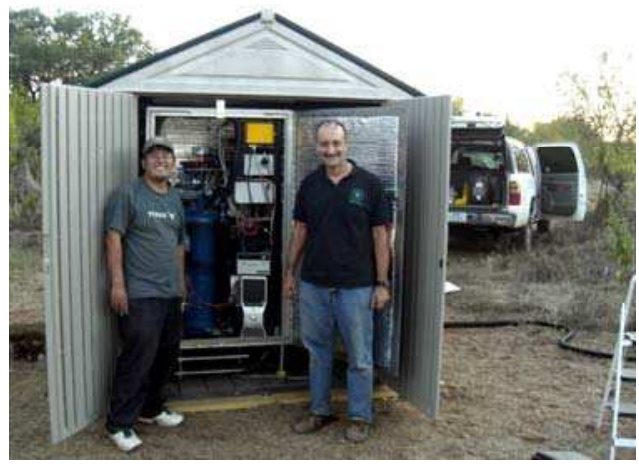

Figure 6. Instrument shed containing E2, during installation November, 2008. A similar shed behind this contains E1. A portable air conditioner in the E1 shed provided cool air for the refrigerator compressor, and was ducted to the E2 shed to cool electronics and the computer. Sheds were covered in reflective insulation to reduce heat load from the sun.

\section{Conclusions}

It is feasible to use the SG as a transportable field instrument with remote unattended operation and wireless internet data transfer and system monitoring. At most sites, a concrete foundation will be required. Even under apparently ideal conditions (outcropping massive limestone) a cemented braced steel rod monument was not sufficiently stable. Climate control is a challenge in a field setting, but can be dealt with using portable cooling equipment. The relatively high drift rate during laboratory and field trials was unrelated to the transportable configuration, and the problem is to be corrected at the GWR factory prior to a forthcoming field experiment in the Arizona desert.

A main motivation for developing a transportable SG is to monitor subsurface fluids. In this role, the SG can serve alone, or support observations with portable relative or absolute gravimeters (Pool and Eychaner, 1995; Naujoks et al 2007). For this purpose, the SG has better precision than the best portable instruments (Kroner and Jahr, 2006), but a multiplicity of local and distant gravity sources make $1 \mu$ gal precision a practical goal. In addition to sensing local fluid storage changes, the SG may be able to identify gravity 'noise', not due to local subsurface fluids. Noise at the level of several to tens of $\mu$ gals occurs over a range of time scales and a variety of sources, including atmospheric attraction not predictable from local barometric pressure, regional and distant water loads, and non-tidal ocean mass redistribution. (Boy and Hinderer, 2006). In support of portable gravimeters, the SG might provide a local 'Gravity Noise Correction' time series similar to an Earth Tide Correction. This would require little understanding of noise sources, and could improve the precision of portable absolute and relative meters when such noise is a limiting factor.

\section{Acknowledgments}

This work was supported by National Science Foundation Instrumentation and Facilities grant EAR03-45864. Additional support provided by the Geology Foundation of the University of Texas at Austin.

\section{References}

Boy J-P and J. Hinderer, 2006, Study of the seasonal gravity signal in superconducting gravimeter data: Journal of Geodynamics, 41, 227-233.

Crossley, D. J., Hinderer, G. Casula, O. Francis, H.-T. Hsu, Y. Imanishi, B. Meurers, J. Neumeyer, B. Richter, K. Shibuya, T. Sato, and T. Van Dam, 1999, Network of superconducting gravimeters benefits a number of disciplines: EOS, Transactions, American Geophysical Union, 80, 121, 125-126.

Goodkind, J. M., 1999, The superconducting gravimeter, Review of Scientific Instruments, 70, 11, 4131-4152.

Hinderrer, J., D. Crossley, and R. Warburton, 2007, Superconducting Gravimeter, Book Chapter 9 in: Treatise on Geophysics, Vol. 3., eds. T. Herring and G. Schubert, Elsevier.

Kroner C and Jahr T, 2006, Hydrological experiments around the superconducting gravimeter at Moxa Observatory. J. of Geodynamics 41;268-275.

Naujoks, M, A. Weise, C. Kroner, and T. Jahr, 2007. Detection of small hydrologic variations in gravity by repeated observations with relative gravimeters, J. Geodesy, December 2007, doi:10.1007/s00190007-0202-9.

Pool D. and J. Eychaner, 1995, Measurements of aquifer storage change and specific yield using gravity surveys: Ground Water, 33, 3, 425-432.

Prothero, W. and J. M. Goodkind, 1968, A superconducting gravimeter: Review of Scientific Instruments, 39, 1257-1262.

Prothero, W. and J. M. Goodkind, 1972, Earth-tide measurements with a superconducting gravimeter: Journal of Geophysical Research, 77, 926-937.

Van Camp M, H. Wenzel, P. Schott P, P. Vauterin, and O. Francis, 2000, Accurate transfer function determination for superconducting gravimeters, Geophysical Research Letters, 27, 37-40. 